\title{
A combination of low-dose bevacizumab and imatinib enhances vascular normalisation without inducing extracellular matrix deposition
}

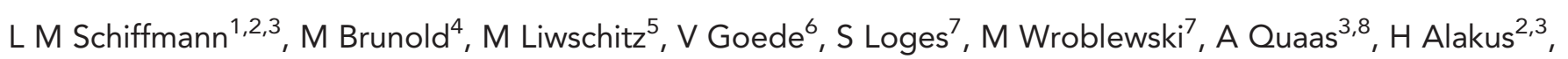

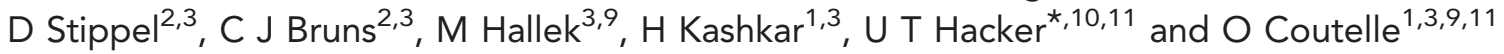

${ }^{1}$ Cologne Excellence Cluster on Cellular Stress Responses in Aging-Associated Diseases (CECAD), Centre for Molecular Medicine Cologne (CMMC) and Institute for Medical Microbiology, Immunology and Hygiene, University of Cologne, CECAD Research Centre, Joseph-Stelzmann-Street 26, 50931 Cologne, Germany; ${ }^{2}$ Department of General, Visceral and Cancer Surgery, University of Cologne, Kerpener Street 62, 50937 Cologne, Germany; ${ }^{3}$ Center for Integrated Oncology (ClO) Cologne-Bonn, Kerpener Street 62, Cologne 50924, Germany; ${ }^{4}$ Department of Internal Medicine III, St Vinzenz Hospital, Merheimer Street 221-223, 50733 Cologne, Germany; ${ }^{5}$ Department of Psychiatry, Psychotherapy and Psychosomatic Medicine, Evangelisches Krankenhaus Bergisch Gladbach, Ferrenbergstr 24, 51465 Bergisch Gladbach, Germany; ${ }^{6}$ Department for Geriatric Medicine, St Marien Hospital, Kunibertskloster 11-13, 50668 Cologne, Germany; ${ }^{7}$ Department of Tumor Biology, Center of Experimental Medicine, University Medical Center Hamburg-Eppendorf, Hamburg, Germany; ${ }^{8}$ Institute for Pathology, University of Cologne, Kerpener Street 62, 50937 Cologne, Germany; ${ }^{9}$ Department I of Internal Medicine, University of Cologne, Kerpener Street 62, 50924 Cologne, Germany and ${ }^{10}$ University Medicine Leipzig, University Cancer Center Leipzig (UCCL), Liebigstr 20, 04103 Leipzig, Germany

Background: Vascular endothelial growth factor (VEGF)-targeting drugs normalise the tumour vasculature and improve access for chemotherapy. However, excessive VEGF inhibition fails to improve clinical outcome, and successive treatment cycles lead to incremental extracellular matrix (ECM) deposition, which limits perfusion and drug delivery. We show here, that low-dose VEGF inhibition augmented with PDGF-R inhibition leads to superior vascular normalisation without incremental ECM deposition thus maintaining access for therapy.

Methods: Collagen IV expression was analysed in response to VEGF inhibition in liver metastasis of colorectal cancer (CRC) patients, in syngeneic (Panc02) and xenograft tumours of human colorectal cancer cells (LS174T). The xenograft tumours were treated with low ( $0.5 \mathrm{mg} \mathrm{kg}^{-1}$ body weight) or high ( $5 \mathrm{mg} \mathrm{kg}^{-1}$ body weight) doses of the anti-VEGF antibody bevacizumab with or without the tyrosine kinase inhibitor imatinib. Changes in tumour growth, and vascular parameters, including microvessel density, pericyte coverage, leakiness, hypoxia, perfusion, fraction of vessels with an open lumen, and type IV collagen deposition were compared.

Results: ECM deposition was increased after standard VEGF inhibition in patients and tumour models. In contrast, treatment with low-dose bevacizumab and imatinib produced similar growth inhibition without inducing detrimental collagen IV deposition, leading to superior vascular normalisation, reduced leakiness, improved oxygenation, more open vessels that permit perfusion and access for therapy.

Conclusions: Low-dose bevacizumab augmented by imatinib selects a mature, highly normalised and well perfused tumour vasculature without inducing incremental ECM deposition that normally limits the effectiveness of VEGF targeting drugs.

${ }^{\star}$ Correspondence: Dr UT Hacker; E-mail: ulrich.hacker@medizin.uni-leipzig.de
${ }^{11}$ These authors share senior authorship.

Received 19 December 2016; accepted 5 January 2017; published online 31 January 2017

(C) 2017 Cancer Research UK. All rights reserved 0007-0920/17 
Since the introduction of the Vascular Endothelial Growth Factor (VEGF)-antibody bevacizumab (Hurwitz et al, 2004), antiangiogenic drugs targeting the VEGF pathway are an integral treatment component for a number of solid tumour entities (Meadows and Hurwitz, 2012). The permanent oversupply of pro-angiogenic factors secreted by tumour and stromal cells induces pathological changes in vessel structure and functionality. As a result, tumour vessels are typically more immature, dysfunctional and leaky compared with their normal blood vessels counterparts. This leads to a raised interstitial fluid pressure (IFP), which reduces perfusion and induces hypoxia, which further stimulates the tumour microenvironment to release more pro-angiogenic cytokines (Jain, 2014).

The elevated IFP in tumours represents a major obstacle for the delivery of anticancer drugs (Heldin et al, 2004). However, pharmacological inhibition of the VEGF pathway can reverse many of these changes both in preclinical models and in cancer patients (Willett et al, 2004; Winkler et al, 2004; Huang et al, 2013). Mechanistically, VEGF inhibitors are thought to 'normalise' the tumour vasculature and lower the IFP by preferentially eliminating immature, leaky blood vessels. Given that normalisation is a requirement for efficient access of chemotherapy (Tong et al, 2004), in a large number of tumour entities, VEGF inhibitors have been most successfully deployed in combination with chemotherapy (Meadows and Hurwitz, 2012).

Another parameter affecting the IFP, which has been linked to tumour progression and metastasis, is the extracellular matrix (ECM) content of tumours (Gilkes et al, 2014). Increased ECM mass is associated with poor drug delivery and has been shown to hinder macromolecules such as immunotherapy from penetrating into the tumour centre (Netti et al, 2000). Conversely, depletion of ECM can improve the delivery of macromolecules and chemotherapy (Brown et al, 2003). Importantly, VEGF withdrawal itself has recently been associated with increased deposition of ECM in malignant tumours (Aguilera et al, 2014). While VEGF inhibition induces the regression of vascular endothelial cells, ECM components such as basement membranes and pericytes accumulate and provide a scaffold for rapid tumour vessel regrowth when the VEGF blockade is interrupted or ineffective (Mancuso et al, 2006).

We have recently demonstrated that such incremental matrix deposition can be avoided by minimizing the dose of the VEGF inhibitor bevacizumab in combination with blocking Ang-2driven angiogenesis in experimental tumour models (Coutelle et al, 2015). The clinical data also indicate that anti-VEGF therapy could adversely affect patient outcome. For example, administration of bevacizumab at $15 \mathrm{mg} \mathrm{kg}^{-1}$ in non-small cell lung cancer patients was associated with reduced perfusion and uptake of chemotherapy (Van der Veldt et al, 2012). Furthermore, in colorectal cancer patients higher bevacizumab doses ( $10 \mathrm{mg} \mathrm{kg}^{-1}$ vs $5 \mathrm{mg} \mathrm{kg}^{-1}$ ) in combination with chemotherapy were found to be less efficient with respect to clinical outcome (Kabbinavar et al, 2003). Yet surprisingly, little attention has been paid to dose reductions of antiangiogenic therapy in clinical practice.

The data from preclinical studies in murine tumour models indicated that inhibition of the platelet-derived growth factor (PDGF) axis can synergize with VEGF blockade (Bergers et al, 2003; Erber et al, 2004). Anti-PDGF therapy has been shown to reduce tumour IFP both as monotherapy (Pietras et al, 2001) and in combination with VEGF blockade (Klosowska-Wardega et al, 2009), translating into improved drug uptake in experimental tumours (Pietras et al, 2002). Notably, synergistic effects on tumour growth have been reported for dual inhibition of VEGF and PDGF in particular, under conditions of submaximal VEGF blockade (Kuhnert et al, 2008).
The tyrosine kinase inhibitor imatinib targets the PDGFReceptor (PDGF-R) signalling axis (Iqbal and Iqbal, 2014) and Abl-kinase, and has proven antiangiogenic efficacy in mouse tumour models as monotherapy or in combination with VEGF inhibition. Imatinib treatment increased drug delivery by lowering the IFP (Pietras et al, 2003; Falcon et al, 2011). In addition, imatinib showed anti-fibrotic effects in mouse models of lung fibrosis (Abdollahi et al, 2005) and breast cancer (Gioni et al, 2008). Here we use low-dose VEGF inhibition augmented with PDGF-R inhibition in a xenograft mouse model to show that this combination leads to superior vascular normalisation without incurring incremental ECM deposition that normally limits the effectiveness of VEGF targeting drugs.

\section{MATERIALS AND METHODS}

Animal tumour models. LS174T human colorectal cancer cells were cultivated as described (Coutelle et al, 2015). Xenograft tumours (LS174T) were generated by subcutaneous injecting of cells $\left(3 \times 10^{6}\right)$ into the flank region of $6-8$-week-old female Balb/ cA nude mice (Charles River, Sulzfeld, Germany). Tumour growth was monitored every other day. After palpable tumour development, mice were randomized into groups of 6 animals each and treatment was initiated. Mice received twice weekly intraperitoneal injections of anti-human VEGF antibody bevacizumab (Roche Pharma, Basel, Switzerland) at $5 \mathrm{mg} \mathrm{kg}^{-1}$ (B100) or $0.5 \mathrm{mg} \mathrm{kg}^{-1}$ body weight (B10) or imatinib mesylate (LC Laboratories, Woburn, MA, USA), dissolved in water (daily intraperitoneally at $70 \mathrm{mg} \mathrm{kg}^{-1}$ body weight), or combinations thereof: B100/Ima or B10/Ima. Tumour volume was calculated as length $\times$ width $^{2} \times \pi / 6$.

A syngeneic subcutaneous mouse tumour model was generated by injection of $5 \times 10^{5}$ murine pancreatic cancer cells (Panc02) into the flank region of 7 -week-old female C57Bl/6J mice. After palpable tumour formation, animals were randomised to receive $20 \mathrm{mg} \mathrm{kg}^{-1}$ DC101 or vehicle i.p. 3 times per week for 14 days. Panc02 cells were cultivated in RPMI 1640 medium ( $10 \%$ FCS, $2 \mathrm{mM}$ L-glutamine, $100 \mathrm{U} \mathrm{ml}^{-1}$ penicillin, $100 \mu \mathrm{g} \mathrm{ml}^{-1}$ streptomycin).

Ethics statement. All mouse studies were performed in accordance with the German animal protection law as approved by local government authorities. Animals were housed in the animal care facility of the University of Cologne or the University Medical Center Hamburg-Eppendorf under standard pathogen-free conditions with a $12 \mathrm{~h}$ light/dark schedule and provided with food and water ad libitum.

Patient samples. Liver metastases were collected at the University Hospital of Cologne with written informed consent from patients with metastatic colorectal cancer concurring with the Declaration of Helsinki. Prior to undergoing surgical resection of liver metastases, the patients had received either chemotherapy plus bevacizumab or chemotherapy alone.

Immunohistochemistry. Murine tumour tissue samples were snap-frozen and prepared for microscopical analyses as described (Coutelle et al, 2015). As primary antibody for staining of endothelial cells, pericytes and type IV collagen, rat monoclonal anti-mouse CD31 antibody (PEACAM-1; 1:50; clone MEC 13.3, BD Pharmingen, Heidelberg, Germany), Cy3conjugated mouse monoclonal anti- $\alpha$-smooth muscle actin antibody $(1: 100 ; \alpha$-smooth-muscle-actin ( $\alpha$-SMA) clone $1 \mathrm{~A} 4$, Sigma-Aldrich, Munich, Germany), rabbit anti-mouse NG2 antibody (1:200; Millipore, Darmstadt, Germany) and rabbit polyclonal anti-type IV collagen antibody ( $1: 100$; Clone ab6586, Abcam, Cambridge, UK) were used, respectively. The CD31 antibody was detected by Alexa Fluor 488-conjugated polyclonal 
goat anti-rat antibody and the NG2 and collagen IV antibody were detected by Alexa Fluor 594-conjugated goat anti-rabbit antibody (1:500; Molecular Probes, Eugene, OR, USA). Immunohistochemical staining of human paraffin-embedded tumour sections was performed using the BOND MAX from Leica (Wetzlar, Germany) according to the protocol of the manufacturers. As a primary antibody Collagen IV clone CIV 22 mouse monoclonal, Dako (M0785; Glostrup, Denmark) at a dilution of $1: 50$ was used.

Vascular leakiness, hypoxia and perfusion studies. To assess vessel leakiness FITC-dextran (MW $2000 \mathrm{kDa}$; Sigma-Aldrich) was used, while for hypoxia studies pimonidazole hydrochloride (Artimmune Analytic GmbH, Kelkheim, Germany) was applied as previously described in detail (Coutelle et al, 2015).

The formation of pimonidazole adducts in hypoxic tumour areas was detected with a FITC monoclonal anti-pimonidazole antibody (1:50; clone 4.3.11.3, Artimmune Analytic $\mathrm{GmbH}$ ). Hoechst 33342 (Sigma-Aldrich) was injected into the tail vein to assess the effect of drug treatment on tumour vessel perfusion as described previously (Coutelle et al, 2015).

Image acquisition and analysis. The MVD representing the number of vessels examined in $\times 100$ images of tumour sections on 5-10 microscopic images per tumour was quantified as previously described (Coutelle et al, 2015). Vessel size was measured using ImageJ software (U.S. National Institutes of Health, Bathesda, Maryland, USA, http://imagej.nih.gov/ij/) in 50 vessels per tumour from representative images of at least three different tumours per group. $\alpha$-SMA pericyte coverage (PC) was quantified from $10-12 \mu \mathrm{m}$-thick $z$ stacks of individual linear vessel stretches with a diameter of at least $6 \mu \mathrm{m}$ in tumour vascular hotspots at $\times 600$ magnification from sections stained for CD31 and $\alpha$-SMA as previously described in detail using ImageJ (Coutelle et al, 2015). NG2 PC was estimated using the ImageJ co-localisation plugin. $\mathrm{PC}$ represents the amount of co-localisation of CD31 and PDGFR- $\beta$ relative to the overall CD31 area density (in $\times 100$ images). Vessel leakiness was analysed in $\times 100$ images of tumours of FITC-dextran-injected mice and the fraction of leaky vessels was determined as previously described.

For oxygen diffusion studies, $\times 40$ multi-image alignments (MIA) of tumour sections from pimonidazole injected mice with CD31 co-staining were generated. The minimal Feret diameter ('halo diameter') of oxygenated areas around vessels (identified by negative pimonidazole immunoreactivity) was determined with ImageJ software, expressing oxygen perfusion as described. The hypoxic tumour fraction was calculated as the percentage of pimonidazole immunoreactivity per whole tumour section, using ImageJ. Non-viable/necrotic tumour areas were identified visually as lacking the proliferation marker Ki67 and showing non- specific or absent staining for 40,6-Diamidin-2-phenylindol (DAPI) and manually outlined for quantification using Adobe Photoshop (Adobe Systems Incorporated, San Jose, CA, USA).

The fraction of blood vessels with an open lumen was determined from 5-10 microscopic images of tumour sections at $\times 100$ magnification from at least three tumours per group. The fraction of open lumen vessels per overall vessel count was calculated. Tumour perfusion with Hoechst-33342 was detected in $\times 100$ images of tumour sections from 3-7 tumours per group. Hoechst immunoreactivity was analysed with ImageJ. Hoechst penetration in closed $v s$ open lumen vessels was quantified by calculating the tissue penetration distance from the vessel margin at different imageJ threshold levels. Relative type IV collagen deposition was estimated by calculating the area density of type IV collagen immunoreactivity per counted vessel (MVD).
Statistical analysis. All results are expressed as average \pm standard error of the mean (s.e.m.). Differences between experimental groups were analysed by unpaired Student's $t$-test or one-way ANOVA for tumour growth curves. $P \leqslant 0.05$ was considered as statistically significant.

\section{RESULTS}

We employed a xenograft tumour model of VEGF-expressing human colorectal cancer cells (LS174T) to evaluate tumour growth in response to PDGF-R blockade in the presence of different degrees of VEGF inhibition by using the VEGF antibody bevacizumab at $5 \mathrm{mg} \mathrm{kg}^{-1}$ (B100) or $0.5 \mathrm{mg} \mathrm{kg}^{-1}$ (B10). PDGF-R was inhibited by the tyrosine kinase inhibitor imatinib either alone (Ima), or in combination with high or low levels of VEGF inhibition (B100/Ima, B10/Ima). VEGF inhibition even at low doses (B10) reduced tumour growth by $50 \%$, whereas tumour growth was not significantly affected by PDGF$\mathrm{R}$ inhibition alone (Ima) compared to the vehicle-treated controls. In contrast, dual inhibition with B10/Ima clearly showed synergistic inhibitory effects with tumour growth being reduced to just $20 \%$ of the controls. A similar level of tumour reduction was otherwise achieved only by applying 10-fold higher doses of either VEGF inhibition (B100) alone or in combination with imatinib (B100/Ima). These findings suggest synergistic effects of imatinib and low-dose bevacizumab (Figure 1A).

Attenuation of tumour growth was accompanied by corresponding changes in the microvascular density (MVD). Whereas PDGF-R inhibition alone (Ima) had little impact on tumour vascularity, low-dose VEGF inhibition (B10) produced a significant reduction in MVD by $55 \%$ compared to controls. The MVD was reduced even further to just $25 \%$ of the controls by dual inhibition with B10/Ima. Comparable levels of reduction in MVD were achieved only by applying 10-fold higher doses of VEGF inhibition (B100) alone or in combination with PDGF-R blockade (B100/Ima; Figure 1B and C). Analysis of the blood vessel size and distribution showed that large diameter vessels $(>400 \mu \mathrm{m})$ were significantly overrepresented in the dual targeting groups B10/Ima and B100/Ima, and to a lesser degree in B10 and B100 groups (Figure 1D) when compared to controls or imatinib treated tumours.

Together, these findings show a remarkable degree of synergism in terms of tumour growth control and vessel reduction for lowdose VEGF inhibition in combination with PDGF-R inhibition (B10/Ima). The data also imply that changes in vessel morphology occur under treatment (Figure 1).

Anti-angiogenic therapy is thought to preferentially eliminate structurally immature leaky blood vessels with poor functionality leading to a lower IFP, which in the clinical setting translates into improved access for conventional chemotherapy. Following antiangiogenic therapy, the remaining mature blood vessels are typically well covered by stabilising pericytes. To assess the maturation state, endothelial cells (CD31) and pericytes ( $\alpha$-SMA) were stained in tumour sections. Blood vessels of control tumours showed an intermediate level of SMA pericyte coverage (54\%) similar to the previously published data (Dang et al, 2008). Compared with controls, no significant change in pericyte coverage was observed upon inhibition of either VEGF (B10, B100) or PDGF-R (Ima) alone. In sharp contrast, when PDGF-R and VEGF inhibition were combined, the pericyte coverage increased significantly: B100/Ima (69\%), B10/Ima (74\%; Figure 2A). Similar results were obtained when the coverage of NG2-positive pericytes was assessed (Supplementary Figure S1). These findings indicate a selection bias towards a more mature vascular phenotype after dual 
A

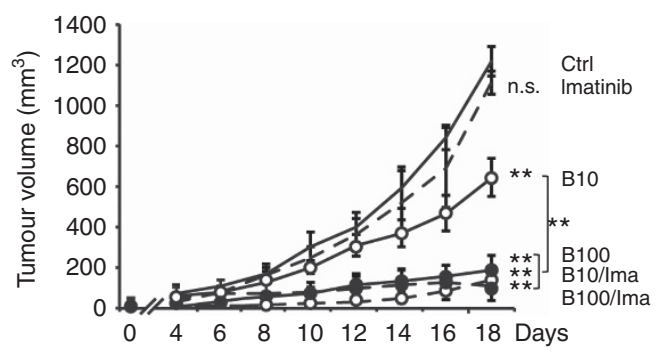

B

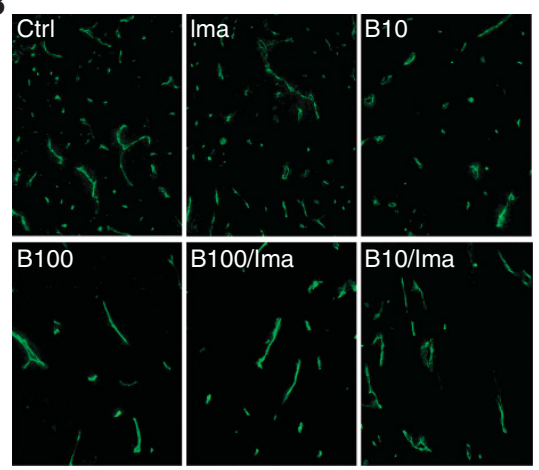

C

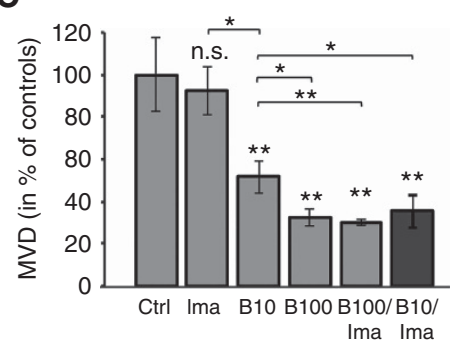

D

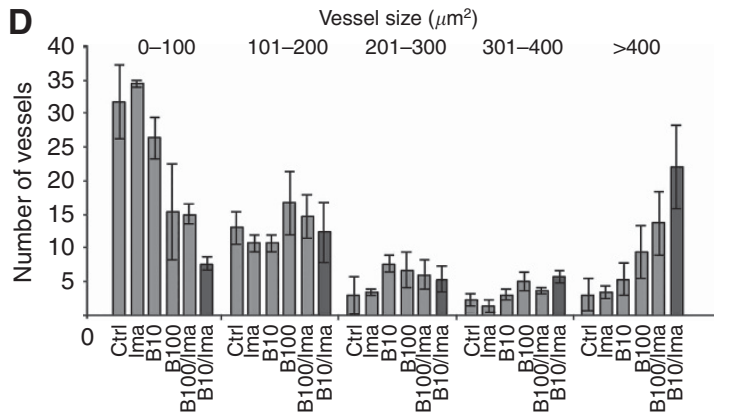

Figure 1. Xenograft tumour growth and vascularity in response to VEGF and PDGF-R inhibition. (A) Tumour growth curves of LS174T xenografts in mice treated with full dose $5 \mathrm{mg} \mathrm{kg}^{-1}$ (B100) or low-dose $0.5 \mathrm{mg} \mathrm{kg}^{-1}$ (B10) bevacizumab or imatinib (and combinations thereof (B10/lma and B100/Ima). (B, C) Quantification of microvessel densitiy (MVD) and (D) vessel size distribution based on CD31-stained endothelial cells in histological tumour sections after 18 days of treatment. Overall, the reduction in MVD in the treatment groups mirrors their growth kinetics. Vessel size distribution is shifted towards larger vessels in the B10/Ima group. Magnification: $\times 100$. Symbols above individual bars indicate statistical significance vs the control group. Symbols above brackets indicate the statistical significance between these groups; ${ }^{\star} P \leqslant 0.05,{ }^{\star \star} P \leqslant 0.01$, n.s. not significant $(P>0.05)$, statistical significance between xenograft growth curves was determined by ANOVA followed by Bonferroni post hoc test.

inhibition. To confirm this, we evaluated vascular integrity by assessing the permeability of the tumour vessels by injecting fluorescent high molecular weight dextran into the tail vein of tumour bearing mice. Nearly all of the blood vessels were leaking dextran in the untreated control tumours, or those subjected to monotherapy with Ima or B10. The fraction of leaky vessels was moderately reduced in the B100 (78\%) and B100/Ima (71\%) groups. In contrast, however, the B10/Ima group showed dextran extravasation in only $37 \%$ of the vessels, confirming a strong selection bias towards mature vessels with intact vascular barrier function. In conclusion, dual inhibition with low-dose bevacizumab and imatinib (B10/Ima) promoted a level of vascular normalisation in the tumours that was not otherwise achievable by VEGF inhibition even at the higher doses typically applied in the clinical setting (Figure 2B).

Given the improvements in barrier function in the B10/Imatreated tumour vasculature, we next investigated if these changes also promoted oxygen penetration into the tumour tissue. To this end, the hypoxic tumour areas were visualised with FITClabelled monoclonal anti-pimonidazole antibody. The distance from the centre of individual blood vessel to the hypoxic rim surrounding them was measured for multiple vessels in each treatment group. Remarkably, local tissue oxygenation was significantly increased in the B10/Ima-treated tumours but not in any of the other treatment groups. The distance from the blood vessel centre to the surrounding hypoxic rim nearly doubled in the B10/Ima tumours compared to the B100 and B100/Ima groups (Figure 3A and B). Furthermore, despite a similar reduction in MVD and similar level of tumour hypoxia, the area of necrosis remained significantly lower in the B10/Ima group. This is consistent with the improved barrier function of the vessels, leading to increased perfusion depth and reduced tissue necrosis (Figure $3 \mathrm{C}$ and $\mathrm{D}$ ). Furthermore, the fraction of open lumen vessels was significantly increased in the B10/Ima group compared with untreated control tumours. The fraction of decompressed (open) vessels nearly doubled in the B10/Ima or imatinib only treatment (Ima) group, whereas VEGF inhibition alone had no significant impact on the fraction of open blood vessels (Figure 4A). To explore if the increase in open lumen vessels was also associated with better access of the fluorescent dye Hoechst-H33342 (H33342)-an established surrogate marker for the delivery of chemotherapy (Morgan et al, 1989)-we intravenously injected $\mathrm{H} 33342$ into tumour-bearing mice. Indeed, H33342 penetration was significantly better in open than compressed vessels (Figure 4C). H33342 perfusion adjusted for vessel density was best in the B10/Ima tumours (Figure 4B).

Increased ECM deposition is thought to lead to elevated mechanical intra-tumoural compression (Jain et al, 2014). As a key ECM component, the type IV collagen immunoreactivity increased dramatically in response to VEGF inhibition: more than two-fold after B10 and more than three-fold after B100 treatment compared to controls (Supplementary Figure S2D and E). Remarkably, the increase in ECM deposition associated with VEGF inhibition could largely be avoided in the B10/Ima group. Although imatinib treatment alone had no effect on type IV collagen deposition, dual inhibition reduced type IV collagen deposition by $50 \%$ in the B10/ Ima group-comparable to untreated controls (Supplementary Figure S2D and E). Together, our findings suggest, that intratumoural matrix deposition can be effectively reduced by low-dose VEGF inhibition in combination with PDGF-R blockade and that the reduced intra-tumoural pressure leads to more open vessels (Figure 4A) and better access for therapeutics (Figure 4B). 
A
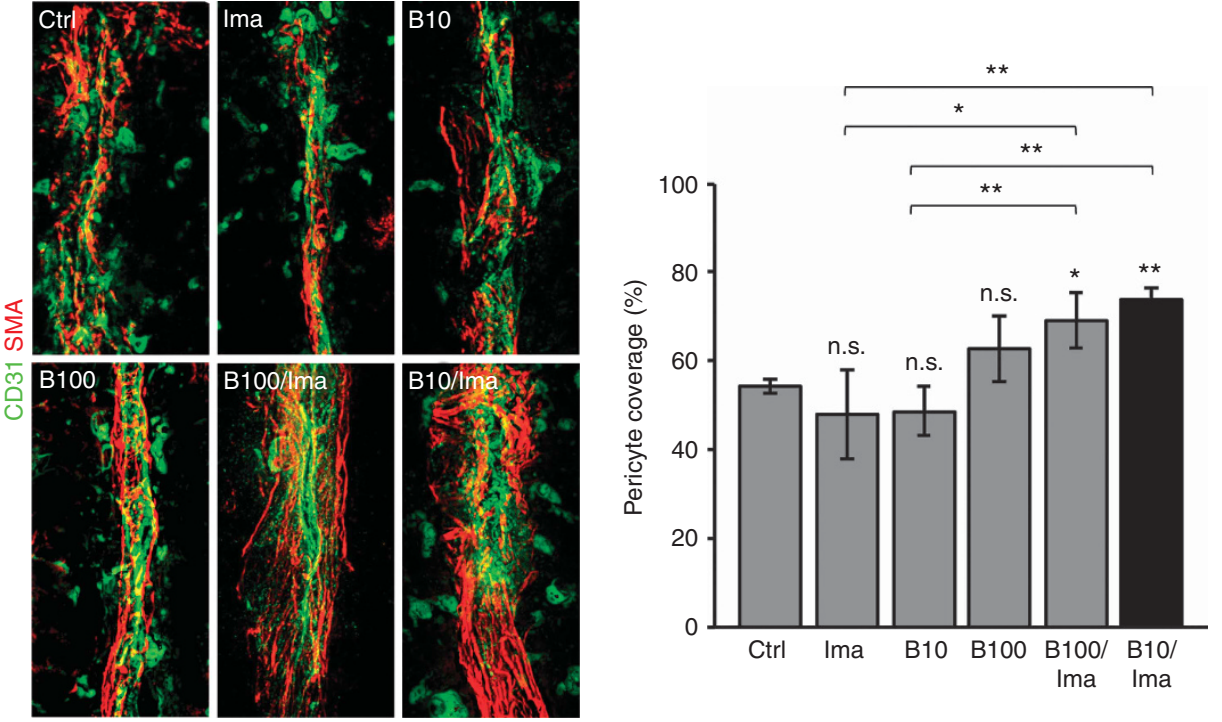

B
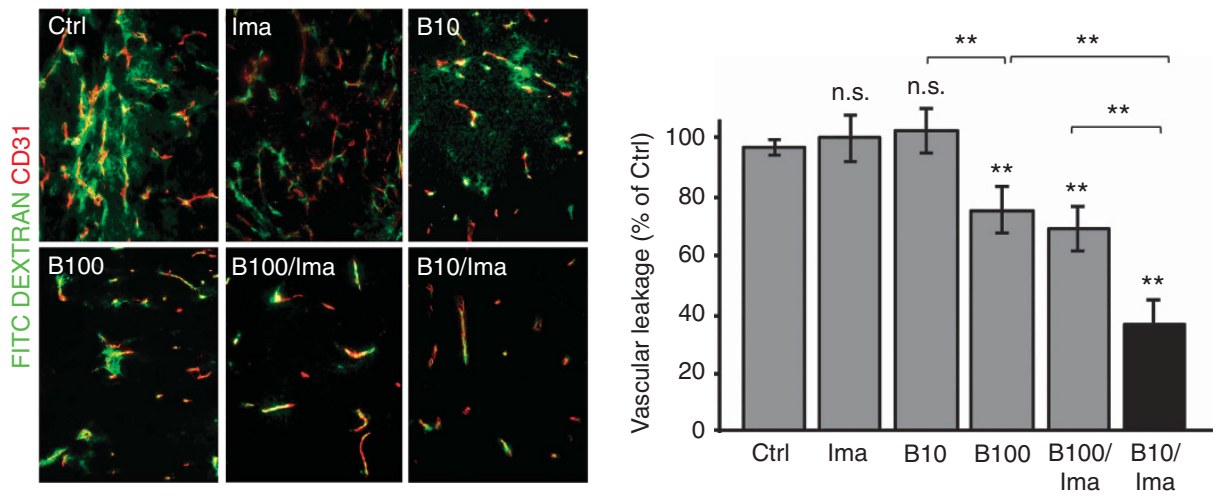

Figure 2. Pericyte coverage and vascular leakage in LS174T tumours. (A) Representative semiconfocal immuno-fluorescence microscopy images of tumour blood vessels (CD31, green) and pericytes (SMA, red) and quantification of pericyte coverage, magnification: $\times 600$. Note the significant increase in pericyte coverage comparing B10 and B10/Ima. (B) Tumour vessels (CD31, red) perfused with FITC-dextran (green) 30 min after tailvein injection and quantification of the fraction of leaky blood vessels in each treatment group, magnification: $\times 100$. Symbols above individual bars indicate statistical significance vs the control group. Symbols above brackets indicate the statistical significance between these respective groups; ${ }^{*} P 0.05,{ }^{\star *} P \leqslant 0.01$, n.s. not significant $(P>0.05)$.

To further substantiate these findings, we carried out a time course analysis of type IV collagen deposition on days 10, 14 and 18 of treatment. The B100 and B100/Ima groups already showed more than twice as much type IV collagen deposition as controls during early treatment stages (day 10), which increased even further by day 18. In contrast collagen IV deposition in the B10/ Ima group started from a much lower early level and continued to decline to the level found in the controls by day 18 of treatment. These data suggest that low-dose VEGF inhibition in combination with imatinib not only limits the initial deposition of type IV collagen but importantly prevents its incremental accumulation during successive treatment cycles of VEGF inhibition thus keeping the tumour accessible for therapy (Figure 5A). To exclude the possibility that type IV collagen deposition following VEGF inhibition is due to the selected xenograft tumour model or the specific anti-VEGF antibody (bevacizumab), we also analysed type IV collagen in resected liver metastasis of colorectal cancer patients $(n=6)$ who were preoperatively treated with conventional chemotherapy in combination with or without bevacizumab. In addition, we analysed collagen IV in a second syngenic mouse pancreatic carcinoma model (Panc02) after treatment with DC101, an antibody directed specifically against murine VEGF-receptor 2. Our analyses show a significant increase in intratumoural type IV collagen following VEGF inhibition, both in the liver metastasis of colorectal cancer patients as well as the Panc02 tumour model (Figure 5B and Supplementary Figure S2A-C). These data provide compelling evidence that VEGF blockade promotes a dosedependent collagen IV deposition in murine tumour models that can be effectively prevented by a combination of low-dose VEGF inhibition and PDGFR inhibition with important clinical implications in colorectal cancer patients.

\section{DISCUSSION}

Vascular endothelial growth factor-targeting drugs preferentially eliminate immature tumour vessels, leading to a reduction in the IFP, improved perfusion and access for therapeutics. However, there is a narrow therapeutic window beyond which excessive VEGF inhibition will result in insufficient perfusion and drug delivery, hypoxia, necrosis and rebound angiogenesis. Clinical evidence supports the concept of the therapeutic window. 
A

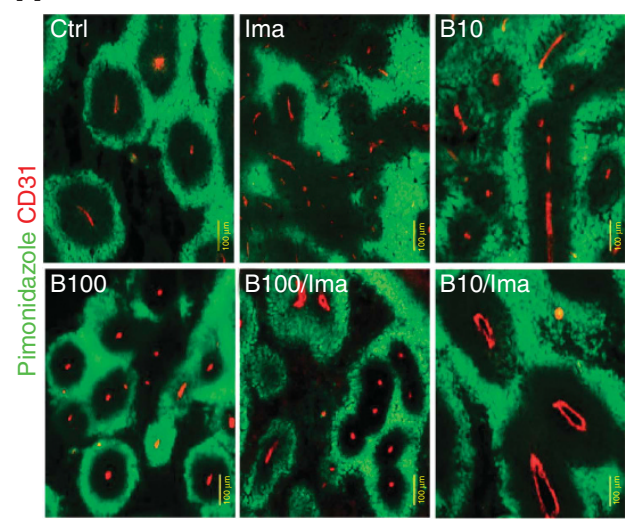

C

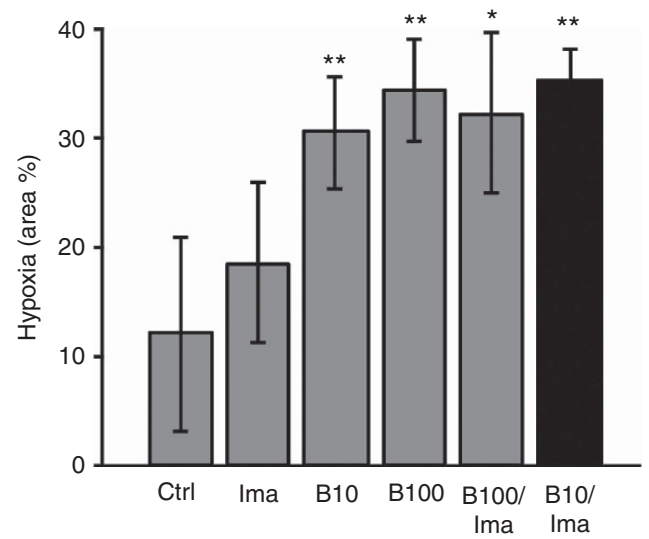

B

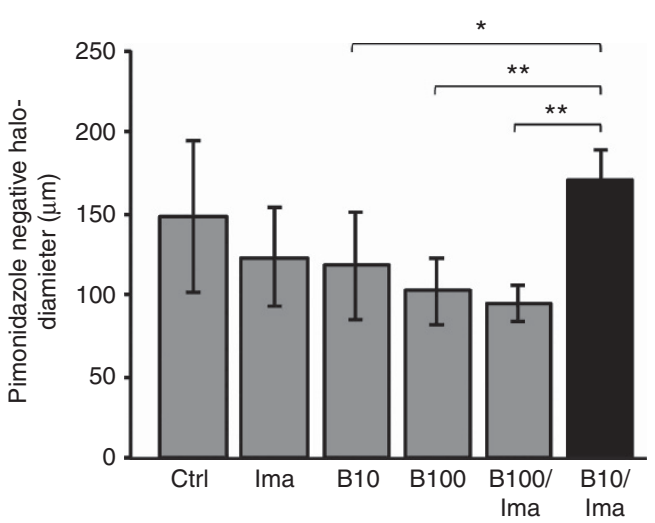

D

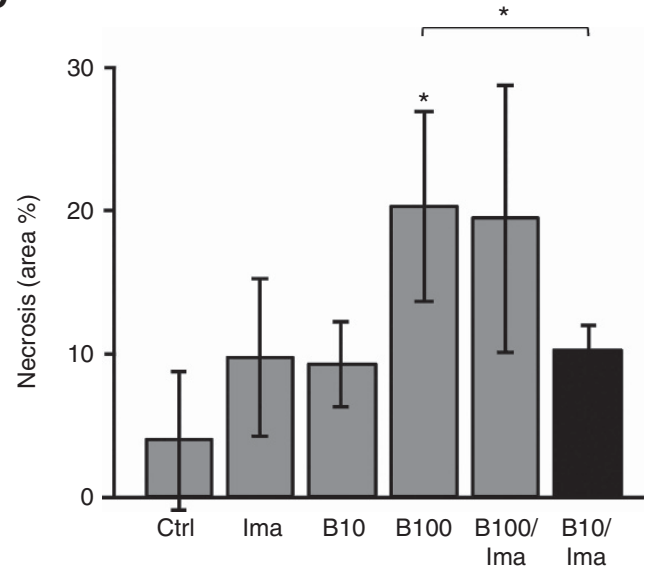

Figure 3. Tumour oxygenation, hypoxia and necrosis in LS174T tumours. (A) Representative immunofluorescence microscopy images of tumour blood vessels (CD31, red) and areas of hypoxia detected by pimonidazole adducts (green), magnification: $\times 100$. (B) Halo-measurement expressing vessel-specific oxygen penetration. (C) Quantification of overall tumour hypoxia (pimonidazole area density) and (D) overall tumour necrosis. Symbols above individual bars indicate statistical significance vs the control group. Symbols above brackets indicate the statistical significance between these two groups; ${ }^{\star} P \leqslant 0.05,{ }^{\star \star} P \leqslant 0.01$. n.s. not significant $(P>0.05)$.

Nevertheless, little attention has been paid to the impact of different levels of VEGF inhibition despite clinical data showing that increasing doses of VEGF inhibition fail to improve clinical outcome (Kabbinavar et al, 2003). Furthermore, effective long term control over tumour growth, requires repeated cycles of chemotherapy and VEGF inhibition, leading to incremental deposition of extracellular matrix components after each treatment cycle. As the work of Mancuso has shown, tumour vessel regression following VEGF inhibition leaves empty sleeves of basement membranes and pericytes that facilitate rapid tumour revascularisation (Mancuso et al, 2006). These accumulating ECM components also contribute to a raised IFP that limits efficient drug delivery, and ultimately drug resistance.

Although multiple tyrosine kinase inhibitors (TKI) such as sunitinib, targeting both VEGF and PDGF-R, have previously been shown to contribute to a lower IFP, higher intra-tumoural blood flow and increased delivery of anti-cancer drugs (Bergers et al, 2003; Erber et al, 2004; Czabanka et al, 2009; Klosowska-Wardega et al, 2009) in clinical trials sunitinib failed to improve outcome in metastatic colorectal cancer patients both as monotherapy (Saltz et al, 2007) or in combination with chemotherapy (Carrato et al, 2013; Hecht et al, 2015). Considering that the fixed ratio of affinities for the respective receptor targets VEGFR and PDGF-R might be suboptimal to achieve overall efficiency, we here used bevacizumab and imatinib to independently control the degree of VEGF and PDGF-inhibition in a xenograft tumour model. Our observation that dual anti-VEGF therapy and PDGF-R inhibition normalises tumour vasculature is not new and corroborates previous reports (Czabanka et al, 2009; Klosowska-Wardega et al, 2009). However, the present study adds to these findings by demonstrating that low-dose VEGF blockade is superior to high-dose VEGF blockade when combined with PDGF-R inhibition in terms of vascular normalisation and ECM matrix deposition. Importantly compared to high doses of VEGF inhibition (B100 and B100/Ima) the combination of B10/Ima selects for a more mature vessel phenotype with improved barrier function and an increase of local tissue oxygenation (Figures 2 and 3). VEGF inhibition has previously been shown to contribute to the accumulation of ECM and to exert pro-fibrotic properties in vitro and in vivo (Kang and Johnson, 2003; Zhang et al, 2015). For example, loss or reduction of VEGF expression in mouse models of pulmonary and renal disease was associated with increased fibrosis in the lung (Stockmann et al, 2010) and renal interstitial fibrosis (Kang and Johnson, 2003). In line with these reports, our data confirm that type IV collagen deposition is increased in response to VEGF inhibition (B100) (Figure 5A). We here show for the first time that this increase in ECM deposition can be avoided by applying the combination of low-dose bevacizumab and imatinib. Critically, this ECM reduction is achieved without compromising vascular normalisation and is accompanied by physical evidence of vascular decompression as illustrated by an increased fraction of blood vessels with an open lumen (Figure 4). Indeed, vascular 

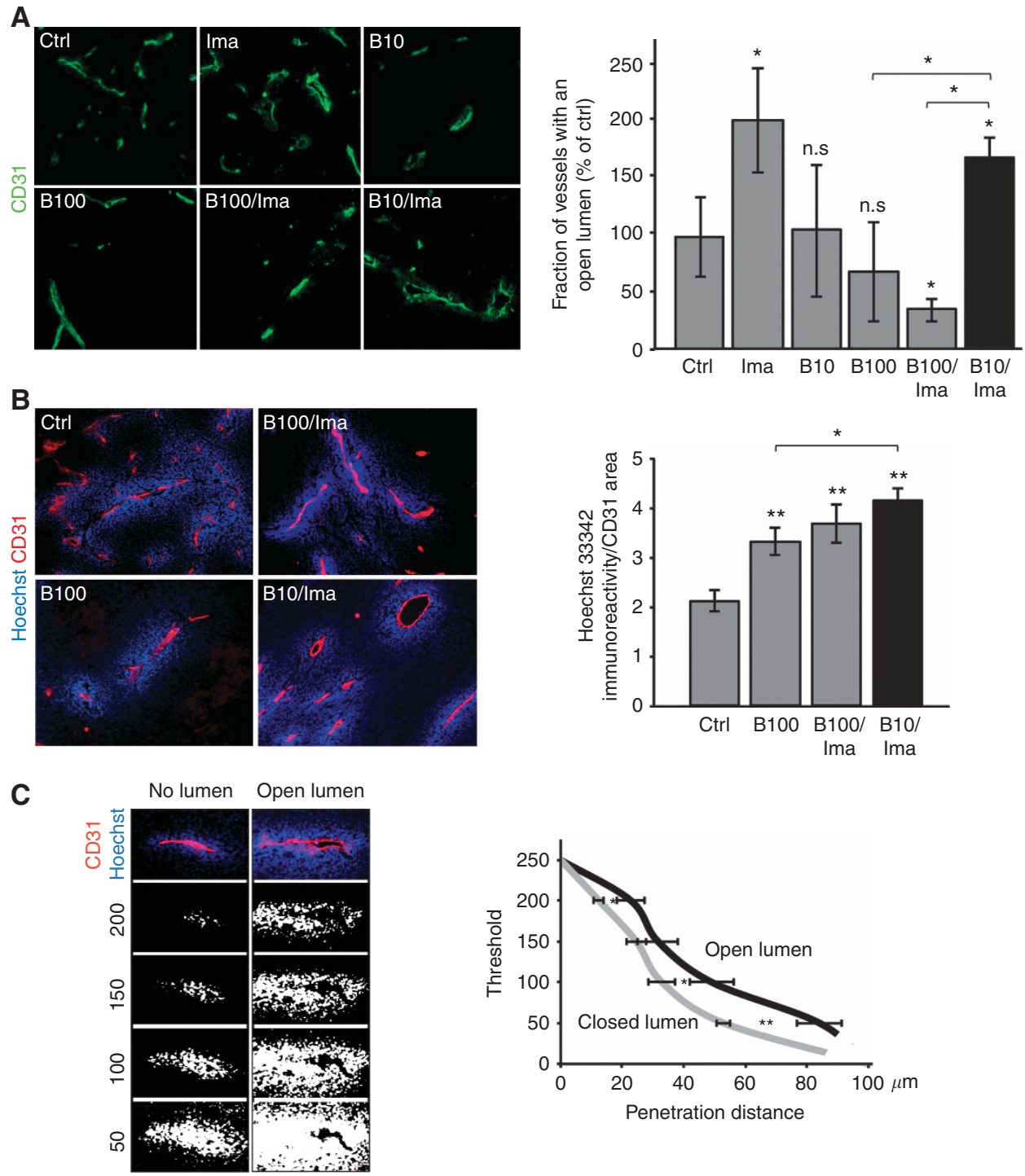

Figure 4. Vessel decompression and Hoechst perfusion in LS174T tumours. (A) Fraction of blood vessels with an open lumen in tumour sections detected with CD31 antibody (green). Both oxygen delivery and the fraction of open lumen vessels is significantly increased following treatment with B10/I. (B) Penetration of tumour tissue with Hoechst-33342 dye (blue) 3 min after tail-vein injection. Blood vessels were stained for CD31 (red). Quantification of Hoechst-33342 area density in cryosections. (C) Hoechst penetration according to vessel lumen status. Hoechst delivery is significantly increased in vessels with an open lumen. Magnification $(\mathbf{A}-\mathbf{C}): \times 100$. Symbols above individual bars indicate statistical significance vs the control group. Symbols above brackets indicate the statistical significance between these two groups; ${ }^{\star} P \leqslant 0.05,{ }^{\star \star} P \leqslant 0.01$. n.s. not significant $(P>0.05)$.

decompression has previously been reported in response to significantly reduced intra-tumoural collagen content (Chauhan et al, 2013). From a therapeutic perspective, it is important to note that the degree of decompression correlates with increased Hoechst-H33342 perfusion (Figure 4B and C), an established surrogate marker for the delivery of chemotherapy (Morgan et al, 1989). Furthermore, the benefit in terms of reduced ECM deposition in the B10/Ima vs B100 treatment group continues to get bigger with successive treatment cycles as our time course analysis shows (Figure 5A). Finally, the deposition of ECM, including type IV collagen in response to VEGF inhibition occurs independently of the tumour model and specific type of VEGF inhibitor used and clearly has physiological relevance. Our data demonstrate that in liver metastases of colorectal cancer patients who received previous bevacizumab-containing treatment type IV collagen deposition was significantly upregulated compared with those that had received chemotherapy only (Figure 5B). This observation highlights the clinical relevance of our preclinical data and encourages further investigation into the potential impact of bevacizumab induced stromal changes in patients.

Given that collagen deposition after VEGF withdrawal represents an important obstacle to effective and long-term therapy, it will be important to further elucidate the interaction between PDGF-R and VEGFR inhibition. Work by Greenberg et al (Greenberg et al, 2008) indicates that in pericytes PDGF-R signalling is inhibited by activated VEGFR through the formation of an inhibitory receptor complex. Conceivably, high (but not low)-dose VEGF inhibition would leave PDGF-R signalling in pericytes unopposed, leading to excessive type IV collagen basement membrane deposition. Whether the Abl-kinase inhibiting activity of imatinib also contributes to the anti-fibrotic effects of this drug combination remains speculative (Aono et al, 2005; Gioni et al, 2008; Akhmetshina et al, 2009).

In summary, the present study provides evidence that reduced doses of anti-VEGF therapy in combination with imatinib can achieve superior levels of vascular normalisation without inducing 
A
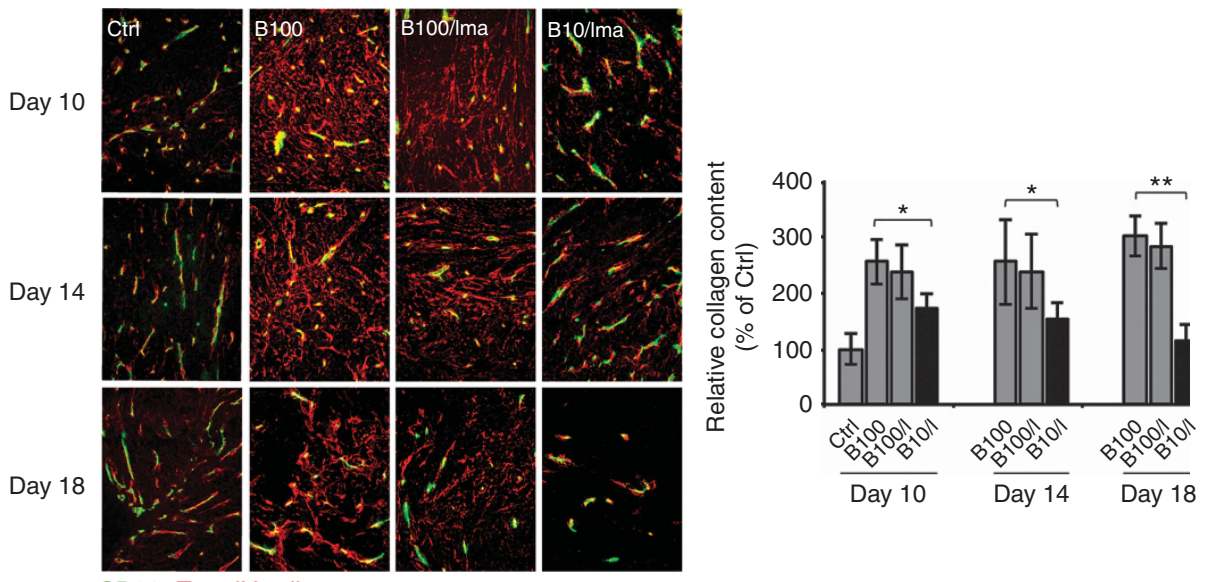

CD31 Type IV collagen

B

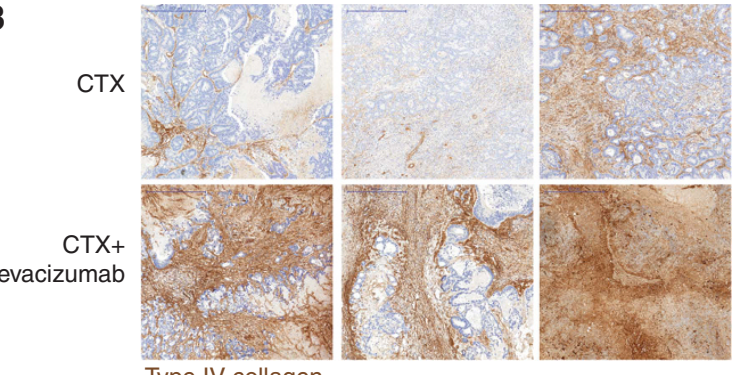

Type IV collagen

Figure 5. Treatment-related changes in extracellular matrix (ECM) deposition in LS174T tumours and patient samples. (A) Time course of type IV collagen expression after 10,14 and 18 days of treatment. Note that any B100-containing treatment groups accumulate type VI collagen during treatment, whereas in the B10/Ima-treated tumours type IV collagen content decreases (B) ECM deposition in resected liver metastases of colorectal cancer patients. Representative images of a resected liver metastasis from three individual patients following neoadjuvant treatment with chemotherapy plus bevacizumab (lower part) compared to representative images of a resected liver metastasis from three individual patients treated with chemotherapy alone (upper part), indicating increased ECM deposition after bevacizumab-containing therapy. Magnification (A):

$\times 100$, (B): scale bar indicates $500 \mu \mathrm{m}$. Symbols above brackets indicate statistical significance between these two groups; ${ }^{\star} P \leqslant 0.05,{ }^{\star} \times P \leqslant 0.01$.

ECM deposition, which is typically observed after VEGF inhibition. Our findings have potential implications for the judicial dosing of VEGF-targeting drugs and their clinical combination with PDGF-R inhibitors, and further highlight the need for radiologic, histologic or serum biomarkers to monitor and individually adjust antiangiogenic therapy.

\section{ACKNOWLEDGEMENTS}

This study was supported by a research grant from the Walther and Marga Boll Foundation, Frechen, Germany. HK and LMS acknowledge funding from the Deutsche Forschungsgemeinschaft (CRU 286 and CRC1218) and the Deutsche Krebshilfe (70112113).

\section{CONFLICT OF INTEREST}

The authors declare no conflict of interest.

\section{REFERENCES}

Abdollahi A, Li M, Ping G, Plathow C, Domhan S, Kiessling F, Lee LB, McMahon G, Grone HJ, Lipson KE, Huber PE (2005) Inhibition of platelet-derived growth factor signaling attenuates pulmonary fibrosis. J Exp Med 201(6): 925-935.

Aguilera KY, Rivera LB, Hur H, Carbon JG, Toombs JE, Goldstein CD, Dellinger MT, Castrillon DH, Brekken RA (2014) Collagen signaling enhances tumor progression after anti-VEGF therapy in a murine model of pancreatic ductal adenocarcinoma. Cancer Res 74(4): 1032-1044.

Akhmetshina A, Venalis P, Dees C, Busch N, Zwerina J, Schett G, Distler O, Distler JH (2009) Treatment with imatinib prevents fibrosis in different preclinical models of systemic sclerosis and induces regression of established fibrosis. Arthritis Rheum 60(1): 219-224.

Aono $\mathrm{Y}$, Nishioka $\mathrm{Y}$, Inayama M, Ugai M, Kishi J, Uehara H, Izumi K, Sone S (2005) Imatinib as a novel antifibrotic agent in bleomycin-induced pulmonary fibrosis in mice. Am J Respir Crit Care Med 171(11): $1279-1285$

Bergers G, Song S, Meyer-Morse N, Bergsland E, Hanahan D (2003) Benefits of targeting both pericytes and endothelial cells in the tumor vasculature with kinase inhibitors. J Clin Invest 111(9): 1287-1295.

Brown E, McKee T, diTomaso E, Pluen A, Seed B, Boucher Y, Jain RK (2003) Dynamic imaging of collagen and its modulation in tumors in vivo using second-harmonic generation. Nat Med 9(6): 796-800.

Carrato A, Swieboda-Sadlej A, Staszewska-Skurczynska M, Lim R, Roman L, Shparyk Y, Bondarenko I, Jonker DJ, Sun Y, De la Cruz JA, Williams JA, Korytowsky B, Christensen JG, Lin X, Tursi JM, Lechuga MJ, Van Cutsem E (2013) Fluorouracil, leucovorin, and irinotecan plus either sunitinib or placebo in metastatic colorectal cancer: a randomized, phase III trial. J Clin Oncol 31(10): 1341-1347.

Chauhan VP, Martin JD, Liu H, Lacorre DA, Jain SR, Kozin SV, Stylianopoulos T, Mousa AS, Han X, Adstamongkonkul P, Popovic Z, Huang P, Bawendi MG, Boucher Y, Jain RK (2013) Angiotensin inhibition enhances drug delivery and potentiates chemotherapy by decompressing tumour blood vessels. Nat Commun 4: 2516.

Coutelle O, Schiffmann LM, Liwschitz M, Brunold M, Goede V, Hallek M, Kashkar H, Hacker UT (2015) Dual targeting of angiopoetin-2 and VEGF potentiates effective vascular normalization without inducing empty basement membrane sleeves in xenograft tumors. Br J Cancer 112(3): 495-503. 
Czabanka M, Vinci M, Heppner F, Ullrich A, Vajkoczy P (2009) Effects of sunitinib on tumor hemodynamics and delivery of chemotherapy. Int $J$ Cancer 124(6): 1293-1300.

Dang DT, Chun SY, Burkitt K, Abe M, Chen S, Havre P, Mabjeesh NJ, Heath EI, Vogelzang NJ, Cruz-Correa M, Blayney DW, Ensminger St WD, Croix B, Dang NH, Dang LH (2008) Hypoxia-inducible factor-1 target genes as indicators of tumor vessel response to vascular endothelial growth factor inhibition. Cancer Res 68(6): 1872-1880.

Erber R, Thurnher A, Katsen AD, Groth G, Kerger H, Hammes HP, Menger MD, Ullrich A, Vajkoczy P (2004) Combined inhibition of VEGF and PDGF signaling enforces tumor vessel regression by interfering with pericyte-mediated endothelial cell survival mechanisms. FASEB J 18(2): 338-340.

Falcon BL, Pietras K, Chou J, Chen D, Sennino B, Hanahan D, McDonald DM (2011) Increased vascular delivery and efficacy of chemotherapy after inhibition of platelet-derived growth factor-B. Am J Pathol 178(6): 2920-2930.

Gilkes DM, Semenza GL, Wirtz D (2014) Hypoxia and the extracellular matrix: drivers of tumour metastasis. Nat Rev Cancer 14(6): 430-439.

Gioni V, Karampinas T, Voutsinas G, Roussidis AE, Papadopoulos S, Karamanos NK, Kletsas D (2008) Imatinib mesylate inhibits proliferation and exerts an antifibrotic effect in human breast stroma fibroblasts. Mol Cancer Res 6(5): 706-714.

Greenberg JI, Shields DJ, Barillas SG, Acevedo LM, Murphy E, Huang J, Scheppke L, Stockmann C, Johnson RS, Angle N, Cheresh DA (2008) A role for VEGF as a negative regulator of pericyte function and vessel maturation. Nature 456: 809-813.

Hecht JR, Mitchell EP, Yoshino T, Welslau M, Lin X, Chow Maneval E, Paolini J, Lechuga MJ, Kretzschmar A (2015) 5-Fluorouracil, leucovorin, and oxaliplatin (mFOLFOX6) plus sunitinib or bevacizumab as first-line treatment for metastatic colorectal cancer: a randomized Phase IIb study. Cancer Manag Res 7: 165-173.

Heldin C-H, Rubin K, Pietras K, Ostman A (2004) High interstitial fluid pressure-an obstacle in cancer therapy. Nat Rev Cancer 4: 806-813.

Huang Y, Stylianopoulos T, Duda DG, Fukumura D, Jain RK (2013) Benefits of vascular normalization are dose and time dependent-letter. Cancer Res 73(23): 7144-7146

Hurwitz H, Fehrenbacher L, Novotny W, Cartwright T, Hainsworth J, Heim W, Berlin J, Baron A, Griffing S, Holmgren E, Ferrara N, Fyfe G, Rogers B, Ross R, Kabbinavar F (2004) Bevacizumab plus irinotecan, fluorouracil, and leucovorin for metastatic colorectal cancer. N Engl J Med 350(23): 2335-2342.

Iqbal N, Iqbal N (2014) Imatinib: a breakthrough of targeted therapy in cancer. Chemother Res Pract 2014: 357027.

Jain RK (2014) Antiangiogenesis strategies revisited: from starving tumors to alleviating hypoxia. Cancer Cell 26(5): 605-622.

Jain RK, Martin JD, Stylianopoulos T (2014) The role of mechanical forces in tumor growth and therapy. Annu Rev Biomed Eng 16: 321-346.

Kabbinavar F, Hurwitz HI, Fehrenbacher L, Meropol NJ, Novotny WF, Lieberman G, Griffing S, Bergsland E (2003) Phase II, randomized trial comparing bevacizumab plus fluorouracil (FU)/leucovorin (LV) with FU/LV alone in patients with metastatic colorectal cancer. J Clin Oncol 21(1): 60-65.

Kang DH, Johnson RJ (2003) Vascular endothelial growth factor: a new player in the pathogenesis of renal fibrosis. Curr Opin Nephrol Hypertens 12(1): 43-49.

Klosowska-Wardega A, Hasumi Y, Burmakin M, Ahgren A, Stuhr L, Moen I, Reed RK, Rubin K, Hellberg C, Heldin CH (2009) Combined anti-angiogenic therapy targeting PDGF and VEGF receptors lowers the interstitial fluid pressure in a murine experimental carcinoma. PLoS One 4(12): e8149.

Kuhnert F, Tam BY, Sennino B, Gray JT, Yuan J, Jocson A, Nayak NR, Mulligan RC, McDonald DM, Kuo CJ (2008) Soluble receptor-mediated selective inhibition of VEGFR and PDGFRbeta signaling during physiologic and tumor angiogenesis. Proc Natl Acad Sci USA 105(29): 10185-10190.
Mancuso MR, Davis R, Norberg SM, O’Brien S, Sennino B, Nakahara T, Yao VJ, Inai T, Brooks P, Freimark B, Shalinsky DR, Hu-Lowe DD, McDonald DM (2006) Rapid vascular regrowth in tumors after reversal of VEGF inhibition. J Clin Invest 116(10): 2610-2621.

Meadows KL, Hurwitz HI (2012) Anti-VEGF therapies in the clinic. Cold Spring Harb Perspect Med 2: a006577.

Morgan SA, Watson JV, Twentyman PR, Smith PJ (1989) Flow cytometric analysis of Hoechst 33342 uptake as an indicator of multi-drug resistance in human lung cancer. Br J Cancer 60(3): 282-287.

Netti PA, Berk DA, Swartz MA, Grodzinsky AJ, Jain RK (2000) Role of extracellular matrix assembly in interstitial transport in solid tumors. Cancer Res 60(9): 2497-2503.

Pietras K, Ostman A, Sjoquist M, Buchdunger E, Reed RK, Heldin CH, Rubin K (2001) Inhibition of platelet-derived growth factor receptors reduces interstitial hypertension and increases transcapillary transport in tumors. Cancer Res 61(7): 2929-2934.

Pietras K, Rubin K, Sjoblom T, Buchdunger E, Sjoquist M, Heldin CH, Ostman A (2002) Inhibition of PDGF receptor signaling in tumor stroma enhances antitumor effect of chemotherapy. Cancer Res 62(19): $5476-5484$.

Pietras K, Stumm M, Hubert M, Buchdunger E, Rubin K, Heldin $\mathrm{CH}$, McSheehy P, Wartmann M, Ostman A (2003) STI571 enhances the therapeutic index of epothilone B by a tumor-selective increase of drug uptake. Clin Cancer Res 9(10): 3779-3787.

Saltz LB, Rosen LS, Marshall JL, Belt RJ, Hurwitz HI, Eckhardt SG, Bergsland EK, Haller DG, Lockhart AC, Rocha Lima CM, Huang X, DePrimo SE, ChowManeval E, Chao RC, Lenz HJ (2007) Phase II trial of sunitinib in patients with metastatic colorectal cancer after failure of standard therapy. J Clin Oncol 25(30): 4793-4799.

Stockmann C, Kerdiles Y, Nomaksteinsky M, Weidemann A, Takeda N, Doedens A, Torres-Collado AX, Iruela-Arispe L, Nizet V, Johnson RS (2010) Loss of myeloid cell-derived vascular endothelial growth factor accelerates fibrosis. Proc Natl Acad Sci USA 107(9): 4329-4334.

Tong RT, Boucher Y, Kozin SV, Winkler F, Hicklin DJ, Jain RK (2004) Vascular normalization by vascular endothelial growth factor receptor 2 blockade induces a pressure gradient across the vasculature and improves drug penetration in tumors. Cancer Res 64(11): 3731-3736.

Van der Veldt AA, Lubberink M, Bahce I, Walraven M, de Boer MP, Greuter HN, Hendrikse NH, Eriksson J, Windhorst AD, Postmus PE,

Verheul HM, Serne EH, Lammertsma AA, Smit EF (2012) Rapid decrease in delivery of chemotherapy to tumors after anti-VEGF therapy: implications for scheduling of anti-angiogenic drugs. Cancer Cell 21(1): 82-91.

Willett CG, Boucher Y, di Tomaso E, Duda DG, Munn LL, Tong RT, Chung DC, Sahani DV, Kalva SP, Kozin SV, Mino M, Cohen KS, Scadden DT, Hartford AC, Fischman AJ, Clark JW, Ryan DP, Zhu AX, Blaszkowsky LS, Chen HX, Shellito PC, Lauwers GY, Jain RK (2004) Direct evidence that the VEGF-specific antibody bevacizumab has antivascular effects in human rectal cancer. Nat Med 10(2): 145-147.

Winkler F, Kozin SV, Tong RT, Chae SS, Booth MF, Garkavtsev I, Xu L, Hicklin DJ, Fukumura D, di Tomaso E, Munn LL, Jain RK (2004) Kinetics of vascular normalization by VEGFR2 blockade governs brain tumor response to radiation: role of oxygenation, angiopoietin-1, and matrix metalloproteinases. Cancer Cell 6(6): 553-563.

Zhang M, Chu S, Zeng F, Xu H (2015) Bevacizumab modulates the process of fibrosis in vitro. Clin Exp Ophthalmol 43(2): 173-179.

This work is published under the standard license to publish agreement. After 12 months the work will become freely available and the license terms will switch to a Creative Commons AttributionNonCommercial-Share Alike 4.0 Unported License.

Supplementary Information accompanies this paper on British Journal of Cancer website (http://www.nature.com/bjc) 